\title{
Improved cardiac function and exercise capacity following correction of pectus excavatum: a review of current literature
}

\author{
Marie Maagaard, Johan Heiberg \\ Department of Cardiothoracic and Vascular Surgery, Aarhus University Hospital, Denmark \\ Correspondence to: Marie Maagaard, MD. Department of Cardiothoracic and Vascular Surgery, Aarhus University Hospital, Palle Juul-Jensens Blv. 99, \\ Dk-8200 Aarhus N, Denmark. Email: maagaard@clin.au.dk.
}

\begin{abstract}
Patients with pectus excavatum (PE) often describe improvements in exercise stamina following corrective surgery. Studies have investigated the surgical effect on physiological parameters; still, no consensus has yet been reached. Therefore, the aim of this literature review was to describe the cardiac outcome after surgical correction, both at rest and during exercise. In February 2016, a detailed search of the databases PubMed, Medline, and EMBASE was performed. We assessed clinical studies that described cardiac outcomes both before and after surgical correction of PE. We only included studies reporting either pre-defined echocardiographic or exercise test parameters. No exclusion criteria or statistical analyses were applied. Twenty-one full-text articles, published between 1972 and 2016, were selected, with cohort-ranges of 3-168 patients, mean age-ranges of 5-33 years, and mean follow-up-ranges from immediately to 4 years after surgery. Twelve studies described resting cardiac parameters. Four studies measured cardiac output, where one described $36 \%$ immediate increase after surgery, one reported $15 \%$ increase after Nuss-bar removal and two found no difference. Three studies demonstrated improvement in mean stroke volume ranges of 22$34 \%$ and two studies found no difference. Fifteen studies investigated exercise capacity, with 11 considering peak $\mathrm{O}_{2}$ pr. $\mathrm{kg}$, where five studies demonstrated improvements with the mean ranging from $8 \%$ to $15 \%$ after surgery, five studies demonstrated no difference, and one saw a decrease of $19 \% 3$ months after Nuss-bar implantation. A measurable increase in exercise capacity exists following surgery, which may be caused by multiple factors. This may be owed to the relief of compressed cardiac chambers with the increased anteriorposterior thoracic dimensions, which could facilitate an improved filling of the heart. With these results, the positive physiological impact of the surgery is emphasized and the potential gain in cardiac function should be integrated in the clinical assessment of patients with $\mathrm{PE}$.
\end{abstract}

Keywords: Pectus excavatum (PE); surgery; cardiac function; echocardiography; exercise

Submitted Mar 07, 2016. Accepted for publication Aug 22, 2016.

doi: $10.21037 /$ acs.2016.09.03

View this article at: http://dx.doi.org/10.21037/acs.2016.09.03

\section{Introduction}

The number of corrective surgeries being performed on pectus excavatum (PE) has increased substantially throughout the last decades (1). The surgical techniques have been refined and high patient satisfaction has been reported (2). Besides the cosmetically pleasing results, the majority of patients also report considerable improvements in physical stamina after surgical correction (3). This has encouraged a suggestion of physiological improvements following surgery, which for many years has been a hot topic among physicians within the field.

This growing interest has prompted a rising number of clinical studies specifically investigating the surgical effect on physiological parameters, but, despite a large amount of studies, no consensus has yet been reached. In literature, the results on pulmonary function are generally inconclusive (4), whereas cardiac parameters tend to point towards improved cardiac function after correction (5). However, most studies include both pulmonary and cardiac parameters and thereby 
the general conclusion on surgical impact is clouded by the diverse pulmonary and cardiac outcomes (5-8). Perhaps for that reason, no clear consensus exists on the indication for operating on the PE patient-is it purely a cosmetic surgery or does the patient also benefit physiologically?

Consequently, we felt the need of a literature review focusing exclusively on cardiac function after surgical correction of $\mathrm{PE}$, both at rest and during exercise.

\section{Methods}

Prior to conducting this literature review, the Cochrane Library was searched to ensure a similar systematic review had not been published. A detailed search of the electronic databases PubMed, Medline, and EMBASE was performed in February 2016 using the following search terms: ("Funnel Chest") AND ("Exercise Test" OR "Treatment Outcome") AND "Humans".

The criteria for inclusion were peer-reviewed, original research, including prospective or retrospective cohort studies, case-control studies, and cross-sectional studies, but not including case reports, non-English language, and publications without a full-text available. No patient age limits were applied. Studies describing patient outcomes after any surgical correction of $\mathrm{PE}$ were included if they also reported any of the following outcome measures: echocardiographic parameters in terms of left ventricular ejection fraction, cardiac output, stroke volume, cardiac index, stroke index, right ventricular end-diastolic volume index or exercise test parameters in terms of peak $\mathrm{O}_{2}$ uptake pr. $\mathrm{kg}, \mathrm{O}_{2}$ pulse, peak stroke index, or peak cardiac index. Since the studies represent a wide variety of different designs and endpoints, no statistical analyses were used. Only the studies demonstrating changes with $\mathrm{P}$ values $<0.05$ were emphasized, whereas trends above a $\mathrm{P}$ value of 0.05 were noted as no change following surgery.

\section{Results}

Based on the above-mentioned criteria and search strategy, we identified 316 records through database searching. Additionally, bibliographies of relevant literature were searched. All records were systematically screened based on their titles and abstracts. Five articles (9-13) met our criteria, but were rejected as they included midterm results for patient-cohorts described in succeeding articles that were included in this review. Ultimately, 21 full-text articles, published between 1972 and 2016, were selected, including a total of 838 patients that were followed pre- and postoperatively. In the included studies, patient numbers ranged from 3 to 168 , with a mean age ranging from 5 to 33 years. Mean follow-up periods in the studies varied from immediately after surgery to 4 years postoperatively, with ranges up to 16 years following surgery. Nine studies used the Nuss procedure; 10 studies described a variety of an open procedure, while one study failed to provide details of the type of surgery. The characteristics of each study can be seen in Table 1.

\section{Cardiac function at rest}

Twelve studies reported on resting cardiac parameters and 10 of them are displayed with results in Table 2. Two studies $(14,15)$ measured other parameters than those shown and were therefore not included in the table.

Six studies $(5,16-20)$ investigated ejection fraction, but only Krueger et al. demonstrated increasing parameters of $14 \%$ immediately after surgery. Four studies (21-24) reported on cardiac output at rest with two describing change following surgery. Chao and colleagues (21) investigated the immediate effect of correction through transesophageal echocardiography and found improvement in right ventricular cardiac output (36\% increase). Sigalet et al. (23) investigated patients after Nuss-bar removal (approximately 39 months follow-up) and found similar changes with a $15 \%$ increase. When adjusting to body surface area by calculating the cardiac index, no studies demonstrated changes at rest following surgery.

Stroke volume was measured by five studies $(19,21-24)$ of which three showed postoperative improvement, ranging from $22 \%$ to $34 \%$, and two found no change. Two additional studies $(14,20)$ adjusted for growth during the study period by calculating the stroke index and reported improvements of $19 \%$ and $69 \%$ after surgical correction. Focusing on the right ventricle, three studies $(5,16,18)$ revealed increasing right ventricular end-diastolic diameters ranging from $10 \%$ to $71 \%$, whereas one study reported no change. Other right ventricular measurements also showed a similar pattern. In two studies by Peterson et al. (20) and Kowalewski et al. (14) right ventricular end-diastolic volume index increased with $40 \%$ and $57 \%$, respectively. Kowalewski et al. furthermore reported an increase of $45 \%$ in right ventricular end-systolic volume following surgery. Huang et al. (15) evaluated the maximum short axis of the right ventricle shortly after the turning of the Nuss-bar and found significant increases both in the transverse distance 


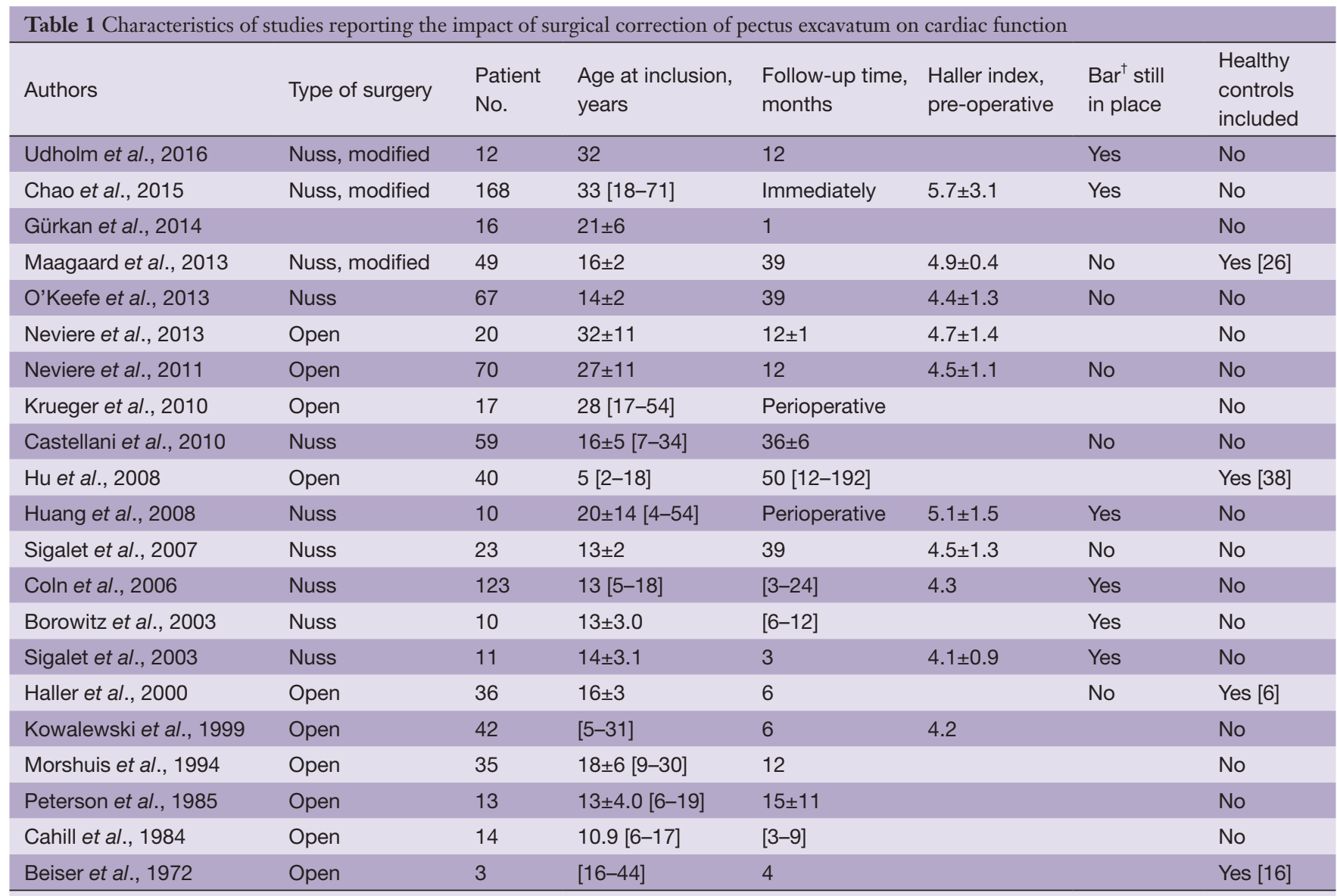

Mean with \pm SD; and in brackets: ranges. Open surgery include: Ravitch, Ravitch-Shamberger, Daniel-technique, and others not specified.

${ }^{\dagger}$ Bar-type: Nuss-bar as well as various types of metal-struts as reported by the studies.

$(15 \%)$ as well as the right ventricular systolic function (16\%). A more recent study (18) looked at right ventricular volume immediately after surgery and found improvement in chamber size $(88 \%)$. Studies $(15,20)$ also evaluated similar parameters of the left ventricle, but found no changes following surgery.

\section{Cardiac function during exercise}

A total of 15 studies investigating exercise before and after operation were identified with 13 displayed in Table 3. Two studies by Beiser et al. (25) and Coln et al. (26) are not listed in the table as they did not provide quantitative measures.

Eleven studies $(5,17,22-24,27-32)$ measured the peak $\mathrm{O}_{2}$ pr. kg in patients before and after surgery. Five studies demonstrated improvements following surgery, with means ranging from $8 \%$ to $15 \%$ and one study (24) found a decrease of $19 \% 3$ months after Nuss-bar implantation.
The remaining studies found no difference before and after surgery.

A total of six studies $(17,22,23,28,31,33)$ reported the $\mathrm{O}_{2}$ pulse which is a surrogate parameter for stroke volume. Five of these found improvements in the follow-up period with means ranging from $6 \%$ to $17 \%$. Two studies determined stroke volume based on the Fick's principle. At our institution, the most recent (5) of the two studies, we found an increase in peak stroke index of $19 \%$, whereas the study from 1985 by Peterson et al. found no change. The cardiac index was measured by three studies $(5,20,27)$, with one demonstrating increases of $23 \%$ following the Nuss-bar removal and two studies displaying no difference following surgery.

\section{Discussion}

The question of a physiological impact from the operation 


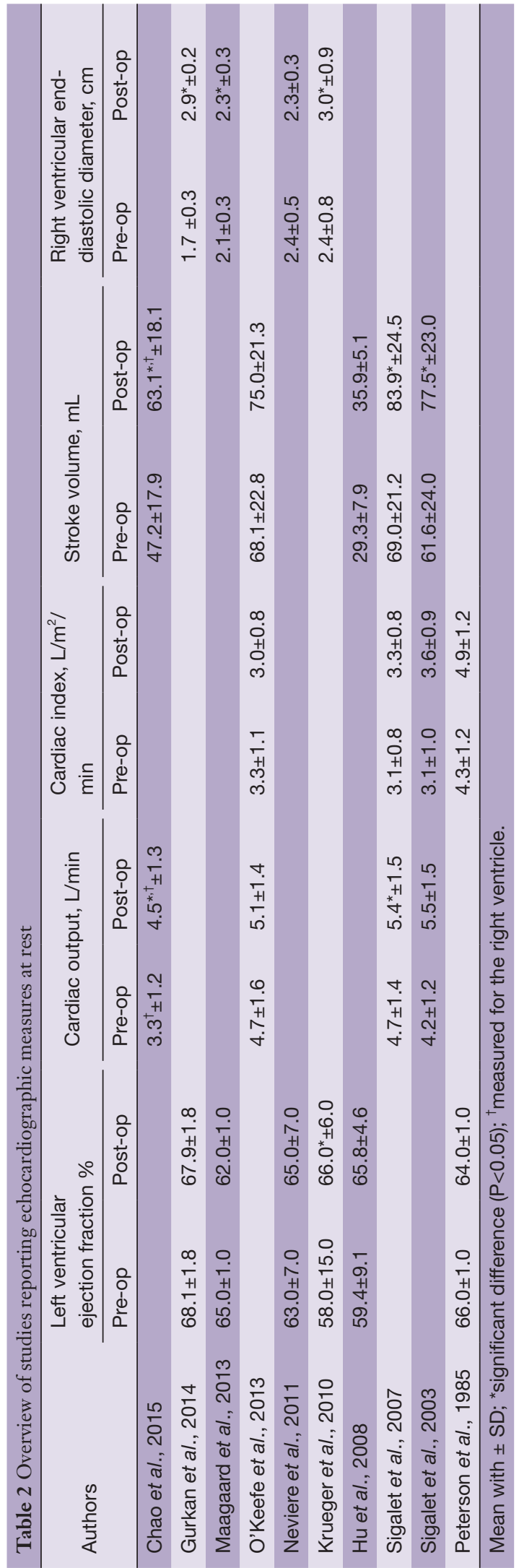

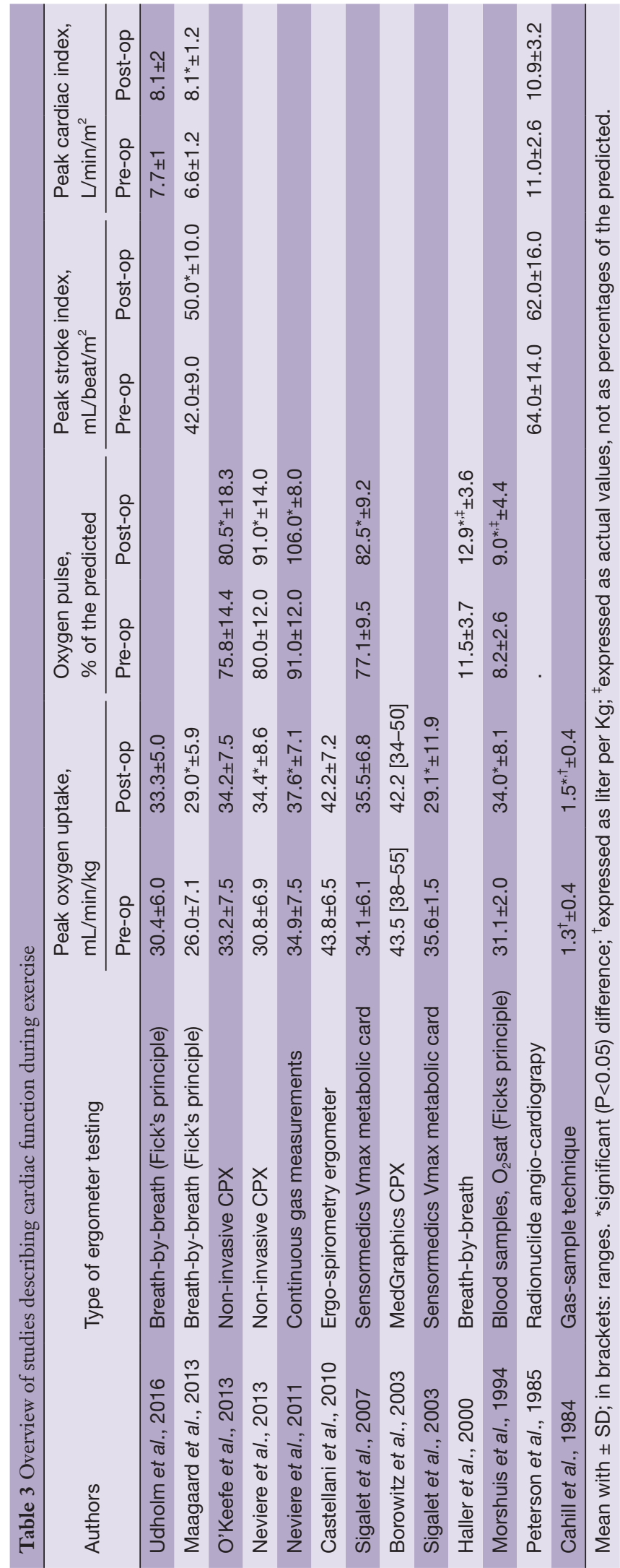


remains a hot topic and no consensus yet exists on the surgical indication for correction of $\mathrm{PE}$. Therefore, our aim was to thoroughly consider cardiac function after surgical correction of PE. The main consensuses are increased right ventricular volumes and, more importantly, increased exercise capacity as an indirect measure of cardiac function.

\section{Cardiac function at rest}

Generally the ejection fraction does not appear to increase following surgery. Only one study (18) demonstrated an increase in left ventricular ejection fraction. Although statistically significant, standard deviations are quite wide and with the chosen technique ejection fraction is estimated only from a single plane. This induces a risk of underestimation prior to surgery because of the geometrically different shape of the ventricles. Possibly, the results are therefore not a reflection of an enhanced left ventricular pumping function, as much as it may be a result of the different positioning of the heart in the thoracic cavity. The other studies reporting on ejection fraction demonstrated deviating tendencies but no others found a similar improvement.

Five studies (19,21-24) evaluated either the cardiac output or the stroke volume following surgery. The most recent study by Chao et al. found a $36 \%$ increase in cardiac output and $34 \%$ increase in stroke volume in 42 patients immediately following the Nuss-procedure. Although the age-range and demographics of this subset of patients were not reported, they were all adults above 18 years of age. In 2007, Sigalet et al. demonstrated an increase of $15 \%$ in cardiac output and $22 \%$ increase in stroke volume following Nuss-bar removal and in 2003 the same research-group found an increase of $26 \%$ in stroke volume. Three other studies $(19,22,24)$ showed the same trends but without significance.

In all the studies reporting on cardiac output and stroke volume, except for Chao et al., the patient cohorts are all in their early teens at inclusion and most of the studies have long-term follow-up. One of the studies (19) even have ageranges from 2.5 to 18 years of age at time of inclusion with follow-up time ranging from 1 year to 16 years. Therefore, the potential physical growth during the study period should be highlighted. The increase depicted in the cardiac volumes following surgery could consequently be attributed solely to the concurrent growth during the study period. One way of addressing this issue is inclusion of healthy, age- and gender-matched controls. However, none of the abovementioned studies considered this.

Another way of attending to the issue of concurrent growth is by using body surface adjusted indices; cardiac index and stroke index. Four studies (20,22-24) considered cardiac index and showed a tendency towards an increase, but without statistical significance. Two older studies $(14,20)$ reported on stroke index following surgical correction and both studies demonstrated a significant improvement. Judging by the results of cardiac index, growth of the young patients may very well play a role in the increasing parameters following surgery. A long-term, follow-up study measuring resting cardiac indices and including an age- and gender-matched control group is needed in order to obtain a better understanding of the resting cardiac parameter. So far, only Chao et al. demonstrated significant improvements, but it would be interesting to see whether these changes could be reproduced with cardiac index and stroke index as the main endpoints.

The two studies by Peterson et al. and Kowalewski et al. both demonstrated increases in right ventricular end-diastolic volume index, which on one side could indicate a degree of cardiac compression relief following surgery. Huang et al. also focused on the right ventricle by measuring the linear dimensions and planar areas and found significant increases both in the short axis and the tricuspid annular plane systolic distance (an index of right ventricular systolic function) in a group of 10 patients immediately after surgery. A more thoroughly evaluated measurement is the end-diastolic diameter of the right ventricle. Four studies $(5,16-18)$ reported an improvement whereas one reported no change. The study by Gurkan et al. investigated 16 adult patients with one-month followup and described a remarkable increase of $47 \%$. However, they only included patients if pre-operative echocardiography revealed right ventricular compression. From Krueger et al. the intraoperative improvements revealed an increase of $25 \%$. Still, the long-term follow-up would be an interesting factor to include in order to observe whether improvements are permanent. In a more recent study from our institution, an increase of $10 \%$ was found in patients after the Nussbar removal, after more than 3 years of follow-up. As the study also included healthy, age-matched controls, the improvement cannot be ascribed the concurrent growth.

Except for the ejection fraction, the majority of the present studies demonstrated an improvement in cardiac resting values following surgery, especially those focusing on the right ventricle. The postoperative increase in stroke volume can be caused by changes both in the geometry 
and position of the right ventricle, as the right ventricle is less concentric and more distensible than the left. Also, the more anterior location in the thoracic cavity of the right ventricle usually places it directly under the sternal depression, making it more exposed to compression preoperatively.

Another explanation could also be that a rise in negative thoracic pressure plays a role in the increased cardiac parameters during rest and especially during exercise. This is supported by Neviere et al. (28), where inspiratory muscle strength was tested before and 1 year after open surgery in 20 adult patients. One year after, inspiratory pressure and static respiratory pressure increased significantly along with exercise $\mathrm{O}_{2}$ pulse and peak $\mathrm{O}_{2}$ pr. kg, suggesting an improvement in the capacity of the inspiratory muscles to produce an intrathoracic negative pressure. The improvement of intrathoracic negative pressure following correction of the chest wall could facilitate improved cardiac filling.

\section{Cardiac function during exercise}

One of the most reported parameters when judging exercise capacity is peak uptake of $\mathrm{O}_{2} \mathrm{pr} . \mathrm{kg}$. Eleven studies reported on this endpoint, with five showing increase. Only one study (24) demonstrated a significant decrease, which could be caused by a very short follow-up period of 3 months after Nuss-bar implantation. With a more sedentary period following surgery, the peak $\mathrm{O}_{2}$ pr. $\mathrm{kg}$ is known to decline somewhat, making it an unreliable measurement at 3 months following the Nuss-procedure.

The tendency of increased exercise capacity is further emphasized when observing stroke volume during exercise, using the indirect measurement of $\mathrm{O}_{2}$ pulse. Six studies report on this, with five showing significant increases ranging from $6 \%$ to $17 \%$. Two of the studies used the Nuss-procedure with follow-up time past bar-removal and the other studies used shorter follow-up time ranging from 6 to 12 months. All studies operated with an open technique. The age of all patients investigated ranged from the mid-teens to early thirties. Even though the surgical technique, patient age, and follow-up time were quite variable, the endpoint still indicates a similar outcome-an improvement in stroke volume during exercise.

Another frequently evaluated parameter is cardiac index during exercise as reported by three studies, all using the Fick's principle. Our group (5) demonstrated a significant improvement of $23 \%$ after Nuss-bar removal, which could not be attributed to growth as a healthy, age-matched control group was also included. Peterson et al. found a slight decrease in the study from 1985, but the study population only consisted of 13 patients with an age range from 6 to 19 and no control group. From 2016, Udholm et al. showed a trending increase in an adult group of patients, however, with a group of only 12 patients and a follow-up time of 1 year, the strength of the study is lacking.

From above, it seems clear that an increase in especially stroke volume during exercise is evident. This is further demonstrated by the small study from Beiser et al., where a group of patients are exercised before and after surgery, both in the supine and in the upright position. Only in the upright position did they show postoperative increase, which is prescribed an increase in stroke volume. This was further supported in 1984 by Cahill and colleagues (32), where an increase in exercise capacity after surgery was found along with significant decrease in heart rate at certain submaximal work-levels. This decrease in heart rate along with concurrent increase in exercise capacity was suggested to be the result of increased cardiac stroke volume. In a study from 2000 by Haller et al. (33), similar findings are reported, where maximal heart rates during the pre- and post-operative exercise study are compared for the same workloads. Here, heart rates are significantly lower during the post-surgical study in patients, along with significant increase in $\mathrm{O}_{2}$ pulse. Control subjects who underwent the tests in the study-period did not show similar changes.

As emphasized, the inclusion of a healthy control group matched for age and gender is of utmost importance in follow-up studies of growing children and teenagers, because of increasing cardiac volumes that occurs naturally with growth, as well as increasing BMI that results from natural, physiological weight. Only three $(5,25,33)$ of 13 studies examining growing teenagers during a follow-up period had included healthy control subjects. Moreover, only two of the studies had the controls matched for age and followed throughout the study period. This should be considered the gold standard when investigating a physiological change through a time period where physiological growth is certain to occur.

Another important factor to include in an exercisestudy is the habitual activity levels of the participants, both patients and control subjects. It has been proposed that the increased exercise values found following surgery could be purely attributed to greater motivation for performing exercise with the correction of the PE and the feeling of improved stamina. Therefore, a questionnaire on physical 
activity habits should be included when investigating exercise capacity during an intervention period, no matter the age-group of the patient cohort. Only three studies $(5,17,28)$ presented a quantitative measurement of daily activity level before and after surgery and showed no difference in physical fitness throughout the study period in accordance to this. Other studies $(14,20,22-25,33)$ reported the perceived exercise tolerance, which does not say much about the actual level of daily activity. Still, they all reported significant improvement, supporting the general knowledge that patients have subjective improvements of ability to perform physical activity.

Only one study (5) investigating growing teenagers during a long-term follow-up period had included healthy, age-matched control-subjects and had quantified the habitual activity level during the study period on all the participants.

\section{Limitations}

Some limitations were present relating to the studies examined. First, the studies varied widely in terms of surgical intervention, number and age of patients included as well as endpoints measured. Furthermore, the interval of the follow-up varied both between the studies, but also often within the same study. Finally, the majority of the studies lack appropriately matched control subjects, thereby considering concurrent growth during the investigational period and also questionnaires investigating the amount of physical activity. Both of these should undeniably be considered in future exercise studies.

\section{Conclusions}

Patients frequently report of improved stamina and reduced symptoms during exercise following the correction of their PE. From this review, it is clear that the increase can be reproduced in the cardiac parameters during exercise. This may be owed to the relief of compressed cardiac chambers with increased anterior-posterior-thoracic dimensions, which again could facilitate an improved filling of the heart. Cardiac resting values with especially those focusing on the right ventricle increased, except for the ejection fraction, which remained unchanged following surgery. From this review, there is a predominance of studies revealing an increased exercise capacity following surgical correction, independent of the surgical approach. Importantly, these potential physiological effects should be integrated in the patient information and reflected in clinical decisionmaking when surgical correction of $\mathrm{PE}$ is considered.

\section{Acknowledgements}

None.

\section{Footnote}

Conflicts of Interest: The authors have no conflicts of interest to declare.

\section{References}

1. Nuss D, Kelly RE Jr. Indications and technique of Nuss procedure for pectus excavatum. Thorac Surg Clin 2010;20:583-97.

2. Jacobsen EB, Thastum M, Jeppesen JH, et al. Healthrelated quality of life in children and adolescents undergoing surgery for pectus excavatum. Eur J Pediatr Surg 2010;20:85-91.

3. Kelly RE Jr, Cash TF, Shamberger RC, et al. Surgical repair of pectus excavatum markedly improves body image and perceived ability for physical activity: multicenter study. Pediatrics 2008;122:1218-22.

4. Kubiak R, Habelt S, Hammer J, et al. Pulmonary function following completion of Minimally Invasive Repair for Pectus Excavatum (MIRPE). Eur J Pediatr Surg 2007;17:255-60.

5. Maagaard M, Tang M, Ringgaard S, et al. Normalized cardiopulmonary exercise function in patients with pectus excavatum three years after operation. Ann Thorac Surg 2013;96:272-8.

6. Jayaramakrishnan K, Wotton R, Bradley A, et al. Does repair of pectus excavatum improve cardiopulmonary function? Interact Cardiovasc Thorac Surg 2013;16:865-70.

7. Kelly RE Jr, Mellins RB, Shamberger RC, et al. Multicenter study of pectus excavatum, final report: complications, static/exercise pulmonary function, and anatomic outcomes. J Am Coll Surg 2013;217:1080-9.

8. Johnson JN, Hartman TK, Pianosi PT, et al. Cardiorespiratory function after operation for pectus excavatum. J Pediatr 2008;153:359-64.

9. Quigley PM, Haller JA Jr, Jelus KL, et al. Cardiorespiratory function before and after corrective surgery in pectus excavatum. J Pediatr 1996;128:638-43.

10. Kowalewski J, Barcikowski S, Brocki M. Cardiorespiratory function before and after operation for pectus excavatum: 
medium-term results. Eur J Cardiothorac Surg 1998;13:275-9.

11. Bawazir OA, Montgomery M, Harder J, et al. Midterm evaluation of cardiopulmonary effects of closed repair for pectus excavatum. J Pediatr Surg 2005;40:863-7.

12. Tang $M$, Nielsen HH, Lesbo M, et al. Improved cardiopulmonary exercise function after modified Nuss operation for pectus excavatum. Eur J Cardiothorac Surg 2012;41:1063-7.

13. Wurtz A, Rousse N, Benhamed L, et al. Simplified open repair for anterior chest wall deformities. Analysis of results in 205 patients. Orthop Traumatol Surg Res 2012;98:319-26.

14. Kowalewski J, Brocki M, Dryjanski T, et al. Pectus excavatum: increase of right ventricular systolic, diastolic, and stroke volumes after surgical repair. J Thorac Cardiovasc Surg 1999;118:87-92; discussion 92-3.

15. Huang PM, Liu CM, Cheng YJ, et al. Evaluation of intraoperative cardiovascular responses to closed repair for pectus excavatum. Thorac Cardiovasc Surg 2008;56:353-8.

16. Gürkan U, Aydemir B, Aksoy S, et al. Echocardiographic assessment of right ventricular function before and after surgery in patients with pectus excavatum and right ventricular compression. Thorac Cardiovasc Surg 2014;62:231-5.

17. Neviere R, Montaigne D, Benhamed L, et al. Cardiopulmonary response following surgical repair of pectus excavatum in adult patients. Eur J Cardiothorac Surg 2011;40:e77-82.

18. Krueger T, Chassot PG, Christodoulou M, et al. Cardiac function assessed by transesophageal echocardiography during pectus excavatum repair. Ann Thorac Surg 2010;89:240-3.

19. Hu TZ, Li Y, Liu WY, et al. Surgical treatment of pectus excavatum: 30 years 398 patients of experiences. J Pediatr Surg 2008;43:1270-4.

20. Peterson RJ, Young WG Jr, Godwin JD, et al. Noninvasive assessment of exercise cardiac function before and after pectus excavatum repair. J Thorac Cardiovasc Surg 1985;90:251-60.

21. Chao CJ, Jaroszewski DE, Kumar PN, et al. Surgical repair of pectus excavatum relieves right heart chamber compression and improves cardiac output in adult patients-an intraoperative transesophageal echocardiographic study. Am J Surg 2015;210:1118-24; discussion 1124-5.

22. O'Keefe J, Byrne R, Montgomery M, et al. Longer term effects of closed repair of pectus excavatum on cardiopulmonary status. J Pediatr Surg 2013;48:1049-54.
23. Sigalet DL, Montgomery M, Harder J, et al. Long term cardiopulmonary effects of closed repair of pectus excavatum. Pediatr Surg Int 2007;23:493-7.

24. Sigalet DL, Montgomery M, Harder J. Cardiopulmonary effects of closed repair of pectus excavatum. J Pediatr Surg 2003;38:380-5; discussion 380-5.

25. Beiser GD, Epstein SE, Stampfer M, et al. Impairment of cardiac function in patients with pectus excavatum, with improvement after operative correction. N Engl J Med 1972;287:267-72.

26. Coln E, Carrasco J, Coln D. Demonstrating relief of cardiac compression with the Nuss minimally invasive repair for pectus excavatum. J Pediatr Surg 2006;41:683-6; discussion 683-6.

27. Udholm S, Maagaard M, Pilegaard H, et al. Cardiac function in adults following minimally invasive repair of pectus excavatum. Interact Cardiovasc Thorac Surg 2016;22:525-9.

28. Neviere R, Benhamed L, Duva Pentiah A, et al. Pectus excavatum repair improves respiratory pump efficacy and cardiovascular function at exercise. J Thorac Cardiovasc Surg 2013;145:605-6.

29. Castellani C, Windhaber J, Schober PH, et al. Exercise performance testing in patients with pectus excavatum before and after Nuss procedure. Pediatr Surg Int 2010;26:659-63.

30. Borowitz D, Cerny F, Zallen G, et al. Pulmonary function and exercise response in patients with pectus excavatum after Nuss repair. J Pediatr Surg 2003;38:544-7.

31. Morshuis WJ, Folgering HT, Barentsz JO, et al. Exercise cardiorespiratory function before and one year after operation for pectus excavatum. J Thorac Cardiovasc Surg 1994;107:1403-9.

32. Cahill JL, Lees GM, Robertson HT. A summary of preoperative and postoperative cardiorespiratory performance in patients undergoing pectus excavatum and carinatum repair. J Pediatr Surg 1984;19:430-3.

33. Haller JA Jr, Loughlin GM. Cardiorespiratory function is significantly improved following corrective surgery for severe pectus excavatum. Proposed treatment guidelines. J Cardiovasc Surg (Torino) 2000;41:125-30.

Cite this article as: Maagaard M, Heiberg J. Improved cardiac function and exercise capacity following correction of pectus excavatum: a review of current literature. Ann Cardiothorac Surg 2016;5(5):485-492. doi: 10.21037/acs.2016.09.03 